\title{
Random Walk Representations of the Heisenberg Model
}

\author{
Joseph G. Conlon ${ }^{1}$ and Jan Philip Solovej ${ }^{2}$ \\ Received January 24, 1991; final February 28, 1991
}

\begin{abstract}
We develop random walk representations for the spin- $S$ Heisenberg ferromagnet with nearest neighbor interactions. We show that the spin- $S$ Heisenberg model is a diffusion with local times controlled by the spin- $S$ Ising model. As a consequence, expectations for the Heisenberg model conditioned on zero diffusion are shown to be Ising expectations.
\end{abstract}

KEY WORDS: Heisenberg model; random walk; Ising model.

\section{INTRODUCTION}

In this paper we develop some random walk representations for the spin- $S$ Heisenberg ferromagnet with nearest neighbor interaction on the integer lattice in $d$ dimensions. The representations parallel corresponding representations for the perfect Bose gas. Hence the Heisenberg model can be thought of as an interacting Bose gas. This has been made more explicit in the paper of Dyson ${ }^{(5)}$ (see also ref. 4).

In our picture one thinks of the free Bose gas as an ensemble of noninteracting random walks. The corresponding picture for the Heisenberg model is of an ensemble of interacting walks. The effect of the interaction is to slow down the diffusion process. One can be more precise and identify the local time for the Heisenberg diffusion. It is given in terms of the spin- $S$ Ising model. In particular, expectations for the spin- $S$ Heisenberg model conditioned on zero diffusion are just spin- $S$ Ising expectations.

Since the effect of diffusion should be to lower critical temperature, one is tempted to conjecture that

$$
T_{c}^{\mathrm{H}}(S) \leqslant T_{c}^{\mathrm{I}}(S)
$$

\footnotetext{
${ }^{1}$ Department of Mathematies, University of Michigan, Ann Arbor, Michigan 48109.

${ }^{2}$ Department of Mathematics, University of Toronto, Toronto M5S 1A1, Canada.
} 
where $T_{c}^{\mathrm{H}}(S)$ denotes the critical temperature of the spin- $S$ Heisenberg model and $T_{c}^{\mathrm{I}}(S)$ the critical temperature of the spin- $S$ Ising model. The inequality (1.1) appears to be unknown even in the classical case $S=\infty$. In that case $T_{c}^{\mathrm{H}}(\infty)$ denotes the critical temperature of the classical Heisenberg model and $T_{c}^{\mathrm{I}}(\infty)$ the critical temperature of the continuous-spin Ising model. It was shown in ref. 2 that $T_{c}^{\mathrm{I}}(\infty)>0$ in dimension $d \geqslant 2$.

Some results of the type (1.1) are known for classical systems. In refs. 1 and 8 it is shown that

$$
T_{c}^{\mathrm{H}}(\infty) \leqslant T_{c}^{\mathrm{I}}(1 / 2)
$$

It is also known from Griffiths inequalities ${ }^{(7)}$ that $T_{c}^{\mathrm{I}}(S)$ is a decreasing function of $S$, whence

$$
T_{c}^{\mathrm{I}}(\infty) \leqslant T_{c}^{\mathrm{I}}(1 / 2)
$$

We also identify the $S \rightarrow \infty$ limits of our random walk representations to obtain a random walk representation for the classical Heisenberg model. This representation for the classical Heisenberg model differs from the Symanzik representation. ${ }^{(3)}$ The Symanzik representation is rotation invariant, whereas our representation depends on the direction of the $S_{Z}$ axis.

\section{RANDOM WALKS ON AN INTEGER LATTICE}

Let $\mathbb{Z}^{d}$ be the integer lattice in $d$ dimensions. For integer $L=1,2, \ldots$, let $A$ be the intersection of $\mathbb{Z}^{d}$ with a "cube" of side $L$,

$$
A=[0, L]^{d} \cap \mathbb{Z}^{d}
$$

We impose periodic boundary conditions on $A$ by identifying endpoints. Then $A$ becomes a $d$-dimensional torus with volume $|A|=L^{d}$. We shall be interested in nearest neighbor random walks on $A$.

We first consider discrete-time random walks of length $l$. Let $\omega(m)$, $m=0,1,2, \ldots, l$, be such a walk with $\omega(0) \in A$ fixed. Then the probability of $\omega, P[\omega]$, is just

$$
P[\omega]=(2 d)^{-1}
$$

Let $A$ be the matrix on $A \times A$,

$$
A\left(R, R^{\prime}\right)= \begin{cases}(2 d)^{-1} & \text { if } R, R^{\prime} \text { nearest neighbors } \\ 0 & \text { otherwise. }\end{cases}
$$


Then, if $P[\mathscr{A} \mid \mathscr{B}]$ denotes the probability of $\mathscr{A}$ conditioned on $\mathscr{B}$, we have

$$
P\left[\omega(l)=R \mid \omega(0)=R^{\prime}\right]=A^{l}\left(R, R^{\prime}\right)
$$

Here $A^{l}$ is the $l$ th power of $A$ as a matrix. Equation (2.4) expresses the Markov property of the process.

Next we introduce a time variable. A bond $b$ of the lattice consists of a directed line segment joining two nearest neighbor lattice sites. To each bond $b$ we associate independent time variables characterized by local times $\tau(b)$. Thus,

$$
P[\text { no jump on the bond } b \text { up to time } t]=\exp [-t / \tau(b)]
$$

From (2.5) it easily follows that the expected time between jumps on $b$ is $\tau(b)$. The exponential distribution (2.5) for the time variables means that all time variables are Markov. Hence they determine a continuous-time random walk $X(t)$ on $A$ which is also Markov. For $\beta>0, R, R^{\prime} \in A$, let $G_{A}\left(\beta, R, R^{\prime}\right)$ be the probability

$$
G_{A}\left(\beta, R, R^{\prime}\right)=P\left[X(\beta)=R \mid X(0)=R^{\prime}\right]
$$

Then the Markov property of $X$ implies the semigroup property,

$$
G_{A}\left(\beta, R, R^{\prime}\right)=\sum_{R^{\prime \prime}} G_{A}\left(\beta-t, R, R^{\prime \prime}\right) G_{A}\left(t, R^{\prime \prime}, R^{\prime}\right), \quad 0<t<\beta
$$

for $G_{A}$. The fact that (2.6) gives a probability implies

$$
\sum_{R} G_{A}\left(\beta, R, R^{\prime}\right)=1
$$

From (2.7) it follows that $G_{A}$ satisfies a differential equation

$$
\begin{aligned}
\partial G_{A} / \partial \beta & =-H(R) G_{A} \\
G_{A}\left(0, R, R^{\prime}\right) & =\delta_{R, R^{\prime}}
\end{aligned}
$$

and $H(R)$ is the infinitesimal generator $H$ of the process acting on the variable $R$. From (2.5), (2.7) one can express $H$ in terms of the local times $\tau(b)$. For a bond $b$ with vertices $R, R^{\prime}$ and in the direction from $R^{\prime}$ to $R$ we write

Then we have

$$
\tau(b)=\tau\left(R, R^{\prime}\right)
$$

$$
H \psi(R)=\left[\sum_{\delta} 1 / \tau(R+\delta, R)\right] \psi(R)-\sum_{\delta}[1 / \tau(R, R+\delta)] \psi(R+\delta)
$$


where the sum over $\delta$ is over the unit vectors generating the lattice. Let $L^{2}(A)$ be the space of complex-valued functions $\psi$ on $A$ with norm $\|\cdot\|$,

$$
\|\psi\|^{2}=\sum_{R \in A}|\psi(R)|^{2}
$$

Then $G_{A}$ is the kernel of the operator $\exp (-\beta H)$ on $L^{2}(A)$,

$$
\exp (-\beta H) \psi(R)=\sum_{R^{\prime} \in A} G_{A}\left(\beta, R, R^{\prime}\right) \psi\left(R^{\prime}\right)
$$

The adjoint $H^{*}$ of $H$ on $L^{2}(\Lambda)$ is given by

$$
H^{*} \psi(R)=\left[\sum_{\delta} 1 / \tau(R+\delta, R)\right] \psi(R)-\sum_{\delta}[1 / \tau(R, R+\delta)] \psi(R+\delta)
$$

From (2.13) it follows that

$$
\exp \left(-\beta H^{*}\right) \psi\left(R^{\prime}\right)=\sum_{R \in A} G_{A}\left(\beta, R, R^{\prime}\right) \psi(R)
$$

The identity (2.3) is then just a reflection of the fact that $H^{*}$ has eigenvalue 0 with corresponding eigenfunction $\psi_{0}(R) \equiv 1$. Finally, one should note that (2.15) implies that $G_{A}$ also satisfies the equation

$$
\begin{aligned}
\partial G_{A} / \partial \beta & =-H^{*}\left(R^{\prime}\right) G_{A} \\
G_{A}\left(0, R, R^{\prime}\right) & =\delta_{R, R^{\prime}}
\end{aligned}
$$

In the case when $\tau(b)=1$ for all bonds $b$ we write $H=H_{0}$, and $H_{0}$ is the negative lattice Laplacian on $A$. From (2.3) it is evident that $H_{0}$ is related to $A$ by the expression

$$
H_{0}=2 d I-2 d A
$$

where $I$ is the identity matrix. Hence we have

$$
\begin{aligned}
P\left[X(\beta)=R \mid X(0)=R^{\prime}\right] & =e^{-2 d \beta} e^{2 d \beta A}\left(R, R^{\prime}\right) \\
& =\sum_{l=0}^{\infty} e^{-2 d \beta} \frac{(2 d \beta)^{l}}{l !} A^{l}\left(R, R^{\prime}\right)
\end{aligned}
$$

Let $N(t)$ be the random variable associated to the time variable (2.5) which gives the number of jumps up to time $t$. Then $N(t)$ is Poisson distributed,

$$
P[N(t)=l]=\exp [-t / \tau(b)][t / \tau(b)]^{l} / l !
$$


Hence from (2.4) and (2.19), Eq. (2.18) is the same as

$$
\begin{aligned}
& P\left[X(\beta)=R \mid X(0)=R^{\prime}\right] \\
& \quad=\sum_{l=0}^{\infty} P[l \text { jumps to time } \beta] P\left[\omega(l)=R \mid \omega(0)=R^{\prime}\right]
\end{aligned}
$$

How to compute the probability (2.6) in the case of nonconstant $\tau(b)$ will be discussed at the end of the next section in a more general setting. Here we wish to point out that it is often useful to think of the case where all $\tau(b) \geqslant 1$ in the following way: The walk $X(t)$ sits at a site $R_{0}$ with a Poisson-distributed waiting time whose expectation is $1 / 2 d$. Then it can jump along a bond $b$ starting at $R_{0}$ with probability $1 / 2 d \tau(b)$, but it can also perform a single-site jump from $R_{0}$ to $R_{0}$ with probability

$$
1-\frac{1}{2 d} \sum_{b} \frac{1}{\tau(b)}
$$

where the sum is over the $2 d$ bonds $b$ starting at $R_{0}$. The probability of not jumping away from $R_{0}$ from time 0 to $t$ can then be found by summing over the number $l$ of single-site jumps, i.e.,

$$
\sum_{l=0}^{\infty} \frac{(2 d t)^{l}}{l !}[\exp (-2 d t)]\left[1-\frac{1}{2 d} \sum_{b} \frac{1}{\tau(b)}\right]^{l}=\exp \left[-\sum_{b} \frac{t}{\tau(b)}\right]
$$

The expression on the right in (2.22) is exactly what we obtain from (2.5).

\section{FREE BOSE GAS}

To each $R$ in the periodic lattice $A$ we associate annihilation and creation operators $a(R), a^{*}(R)$, where

$$
\left[a\left(R^{\prime}\right), a^{*}(R)\right]=\delta_{R, R^{\prime}}
$$

The number operator $\mathscr{N}_{A}$ is then given by

$$
\mathscr{N}_{A}=\sum_{R \in A} a^{*}(R) a(R)
$$

The Hamiltonian for the free Bose gas is

$$
\mathscr{H}_{A, B}=\frac{1}{2} \sum_{R \in A, \delta}\left[a^{*}(R+\delta)-a^{*}(R)\right][a(R+\delta)-a(R)]
$$


where the $\delta$ summation is as in (2.11). For any $h>0$, the finite-volume free energy of the Bose gas at inverse temperature $\beta$ is given by

$$
f_{A, B}(\beta, h)=|A|^{-1} \log \left[\operatorname{Tr} \exp \left(-\beta \mathscr{H}_{A}-h \mathcal{N}_{A}\right)\right]
$$

and the thermodynamic free energy by

$$
f_{B}(\beta, h)=\lim _{A \rightarrow \infty} f_{A, B}(\beta, h)
$$

One can obtain formulas for $f_{A, B}, f_{B}$ by going to Fourier variables. Here we wish to obtain a random walk representation which can be compared to a similar representation for the Heisenberg model. To do this we compute the Hamiltonian $\mathscr{H}_{A}$ acting on $N$-particle states. For any set of $N$ points $\left\{R_{1}, \ldots, R_{N}\right\}$ with $R_{i} \in A, 1 \leqslant i \leqslant N$, let $\left|R_{1}, R_{2}, \ldots, R_{N}\right\rangle$ be the state

$$
\left|R_{1}, \ldots, R_{N}\right\rangle=(N !)^{-1 / 2} \prod_{i=1}^{N} a^{*}\left(R_{i}\right)|0\rangle
$$

where $|0\rangle$ is the vacuum. The states $\left|R_{1}, \ldots, R_{N}\right\rangle$ have normalization

$$
\left\langle R_{1}, \ldots, R_{N} \mid R_{1}, \ldots, R_{N}\right\rangle=\prod_{R \in A} n_{R} ! / N !
$$

where

$$
n_{R}=n_{R}\left(R_{1}, R_{2}, \ldots, R_{N}\right)=\#\left\{i: R_{i}=R\right\}, \quad R \in A
$$

The state (3.6) determines the integers $n_{R}$ in (3.8) uniquely. Conversely, a set of nonnegative integers $n_{R}, R \in A$, defines a state (3.6) which is determined up to normalization. We denote the normalized state determined by the integers $n_{R}, R \in A$, by $\left|n_{R}: R \in A\right\rangle$. Thus,

$$
\begin{aligned}
\operatorname{Tr} \exp \left(-\beta \mathscr{H}_{A, B}-h \mathscr{N}_{A}\right)= & \prod_{R \in A} \sum_{n_{R}=0}^{\infty} \exp \left(-h \sum_{R \in A} n_{R}\right) \\
& \times\left\langle n_{R}: R \in A\left|\exp \left(-\beta \mathscr{H}_{A, B}\right)\right| n_{R}: R \in A\right\rangle
\end{aligned}
$$

Next we define a unitary transformation from the $N$-particle space $\mathcal{N}_{A}=N$ to the space $L_{\text {sym }}^{2}\left(\Lambda^{N}\right)$ of symmetric functions $\psi$ on $A^{N}$. For any symmetric function $\psi$ we associate a state $|\psi\rangle$ by

$$
|\psi\rangle=\sum_{R_{i} \in A, 1 \leqslant i \leqslant N} \psi\left(R_{1}, R_{2}, \ldots, R_{N}\right)\left|R_{1}, R_{2}, \ldots, R_{N}\right\rangle
$$


It follows from (3.7) that

$$
\langle\psi \mid \psi\rangle=\sum_{R_{i} \in A, 1 \leqslant i \leqslant N}\left|\psi\left(R_{1}, \ldots, R_{N}\right)\right|^{2}
$$

Equation (3.11) shows that the mapping is unitary. Furthermore, we also have

$$
\mathscr{H}_{A}|\psi\rangle=\sum_{R_{i} \in A, 1 \leqslant i \leqslant N} H_{\Lambda, N} \psi\left(R_{1}, \ldots, R_{N}\right)\left|R_{1}, \ldots, R_{N}\right\rangle
$$

where

$$
H_{\Lambda, N}=\sum_{i=1}^{N} H_{0}\left(R_{i}\right)
$$

and $H_{0}\left(R_{i}\right)$ is the operator (2.17) acting on the variable $R_{i}$. In view of the fact that the transformation is unitary, we have

$$
\operatorname{Tr} \exp \left(-\beta \mathscr{H}_{\Lambda, B}-h \mathscr{N}_{R}\right)=\sum_{N=0}^{\infty} e^{-h N} \operatorname{Tr}_{\mathrm{sym}} \exp \left(-\beta H_{A, N}\right)
$$

where $\operatorname{Tr}_{\text {sym }}$ denotes that the trace is over $L_{\text {sym }}^{2}\left(A^{N}\right)$.

Let $G_{A, N}\left(\beta, \mathbf{R}, \mathbf{R}^{\prime}\right)$ be the Green's function for the operator $H_{A, N}$ on $L^{2}\left(\Lambda^{N}\right)$. Thus, if $\psi(\mathbf{R})=\psi\left(R_{1}, R_{2}, \ldots, R_{N}\right), R_{i} \in \Lambda, 1 \leqslant i \leqslant N$, is a function on $A^{N}$, then

$$
\exp \left(-\beta H_{A, N}\right) \psi(\mathbf{R})=\sum_{R^{\prime} \in \Lambda^{N}} G_{A, N}\left(\beta, \mathbf{R}, \mathbf{R}^{\prime}\right) \psi\left(\mathbf{R}^{\prime}\right)
$$

We can express the trace in (3.14) in terms of the Green's functions $G_{A, N}$ by choosing an orthonormal basis for $L_{\text {sym }}^{2}\left(\Lambda^{N}\right)$ and using (3.15). A convenient basis is the image of the basis $\left|n_{R}: R \in A\right\rangle$ under the unitary transformation (3.10). We denote this basis by $\mid n_{R}: R \in A$ ). It is easy to see that

where

$$
\left.\mid n_{R}: R \in A\right)=\psi\left(x_{1}, \ldots, x_{N}\right)
$$

$\psi\left(x_{1}, \ldots, x_{N}\right)=\left(\prod_{R \in A} n_{R} ! / N !\right)^{1 / 2} \quad$ if $\quad n_{R}=\#\left\{i: x_{i}=R\right\}, \quad R \in A$

$\psi\left(x_{1}, \ldots, x_{N}\right)=0 \quad$ otherwise

For $R \in A$ let $\delta_{R}(x)$ be the $\delta$ function

$$
\delta_{R}(x)= \begin{cases}1 & \text { if } x=R \\ 0 & \text { otherwise }\end{cases}
$$


Then the function $\psi$ in (3.16) can be written in terms of $\delta$ functions. Let $R_{1}, \ldots, R_{N}$ be a set of $N$ points satisfying (3.8). Then

$\psi\left(x_{1}, \ldots, x_{N}\right)=\left(\prod_{R \in A} n_{R} ! / N !\right)^{1 / 2} \prod_{R \in A}\left(1 / n_{R} !\right) \sum_{\pi} \delta_{R_{\pi 1}}\left(x_{1}\right) \cdots \delta_{R_{\pi N}}\left(x_{N}\right)$

where the sum in (3.18) is over all permutations $\pi$ on $\{1,2, \ldots, N\}$. From (3.15) and (3.18) we obtain then the formula

$$
\left(n_{R}: R \in A\left|\exp \left(-\beta H_{A, N}\right)\right| n_{R}: R \in \Lambda\right)=\prod_{R \in A}\left(1 / n_{R} !\right) \sum_{\pi} G_{A, N}\left(\beta, \mathbf{R}, \mathbf{R}_{\pi}\right)
$$

where

$$
\mathbf{R}=\left(R_{1}, R_{2}, \ldots, R_{N}\right), \quad \mathbf{R}_{\pi}=\left(R_{\pi 1}, R_{\pi 2}, \ldots, R_{\pi N}\right)
$$

From (3.19) it follows then that the formula for the trace is given by

$$
\operatorname{Tr}_{\text {sym }} \exp \left(-\beta H_{A, N}\right)=\frac{1}{N !} \sum_{\mathbf{R} \in \Lambda^{N}, \pi} G_{A, N}\left(\beta, \mathbf{R}, \mathbf{R}_{\pi}\right)
$$

It is straightforward now to obtain a formula for the free energy (3.4) if we use the fact that

$$
G_{\Lambda, N}\left(\beta, \mathbf{R}, \mathbf{R}^{\prime}\right)=\prod_{i=1}^{N} G_{\Lambda}\left(\beta, R_{i}, R_{i}^{\prime}\right)
$$

where $G_{A}$ is the one-particle Green's function satisfying (2.9) with $H=H_{0}$. For a given permutation $\pi$ on $\{1,2, \ldots, N\}$ let $m_{j}, j=1,2, \ldots$, be the number of cycles of length $j$. Then we must have

$$
\sum_{j=1}^{\infty} j m_{j}=N
$$

For given values $m_{j}$ satisfying (3.23) the number of such permutations $\pi$ is

$$
N ! / \prod_{j=1}^{\infty}\left(j^{m_{j}} m_{j} !\right)
$$

From (3.21), (3.22), and (3.24) and the semigroup property for $G_{A}$ it follows then that

$$
\operatorname{Tr}_{\mathrm{sym}} \exp \left(-\beta H_{A, N}\right)=\sum_{m_{j} \geqslant 0, j=1,2, \ldots} \prod_{j=1}^{\infty}\left[\frac{1}{j} \sum_{R \in A} G_{A}(j \beta, R, R)\right]^{m_{j}} / m_{j} !
$$


where the $m_{j}$ satisfy the constraint (3.23). From (3.14) it then follows that

$$
\begin{aligned}
\exp \left[|\Lambda| f_{A, B}(\beta, h)\right] & =\operatorname{Tr} \exp \left(-\beta \mathscr{H}_{\Lambda, B}-h \mathcal{N}_{A}\right) \\
& =\exp \left[\sum_{j=1}^{\infty} \frac{e^{-j h}}{j} \sum_{R \in A} G_{A}(j \beta, R, R)\right]
\end{aligned}
$$

Since $H_{A, N} \geqslant 0$, the expression in (3.19) is less than 1. We wish to make this explicit by writing (3.19) as a probability. To each bond $b$ in $A$ we can associate a unique lattice point, namely the origin of the vector $b$. Thus if $b=\overrightarrow{R^{\prime} R}$, then the lattice point is $R^{\prime}$. We can then define $n(b)$ by

$$
n(b)=n_{R^{\prime}} \text {, the number of particles at } R^{\prime}
$$

For an arbitrary bond $b$ we can also define a differential $d b(x)$ which is a function of $x \in A$. For the bond $b=\overrightarrow{R^{\prime} R}, d b$ is given by

$$
d b(x)=\left\{\begin{aligned}
-1 & \text { if } x=R^{\prime} \\
+1 & \text { if } x=R \\
0 & \text { otherwise }
\end{aligned}\right.
$$

In analogy to (3.27), we shall also write $d b\left(b^{\prime}\right)=d b\left(x^{\prime}\right)$ for the bond $b^{\prime}=\overrightarrow{x^{\prime} x}$. Using this notation, we can write (3.19) as a probability. Let $\left(b_{1}, b_{2}, \ldots, b_{m}\right)$ be a sequence of bonds in $A$. We shall say this sequence is compatible with the state $\left.\mid n_{R}: R \in A\right)$, written $\left.\left(b_{1}, \ldots, b_{m}\right) \sim \mid n_{R}: R \in A\right)$, if

$$
\begin{aligned}
n(x)+\sum_{i=1}^{k} d b_{i}(x) \geqslant 0, & \text { all } \quad x \in A, \quad 1 \leqslant k \leqslant m \\
\sum_{i=1}^{m} d b_{i}(x) & =0, \quad \text { all } \quad x \in A
\end{aligned}
$$

For given local times $\tau(b)$, particle numbers $n_{R}, R \in A$, and sequence $\left(b_{1}, \ldots, b_{m}\right)$ we define the probability of the sequence

$$
\begin{aligned}
P_{\beta}\left[\left(b_{1},\right.\right. & \left.\left.b_{2}, \ldots, b_{m}\right) ; n_{R}, R \in A\right] \\
= & \int_{0=t_{0}<t_{1}<\cdots<t_{m}<\beta} d t_{1} \cdots d t_{m} \\
& \times \prod_{i=1}^{m} \prod_{b \neq b_{i}} \exp \left\{-\left[n(b)+\sum_{j=1}^{i-1} d b_{j}(b)\right] \frac{t_{i}-t_{i-1}}{\tau(b)}\right\} \\
& \times\left(-\frac{d}{d t_{i}}\right) \exp \left\{-\left[n(b)+\sum_{j=1}^{i-1} d b_{j}\left(b_{i}\right)\right] \frac{t_{i}-t_{i-1}}{\tau\left(b_{i}\right)}\right\} \\
& \times \prod_{b} \exp \left[-n(b) \frac{\beta-t_{m}}{\tau(b)}\right]
\end{aligned}
$$


Equation (3.30) is the probability that a particle jumps along $b_{1}$, then another along $b_{2}$, until finally a last particle jumps along $b_{m}$. We can see then from (3.19) that if we take $\tau(b)=1$ for all $b$, that

$$
\begin{aligned}
\left(n_{R}:\right. & \left.R \in A\left|\exp \left(-\beta H_{A, N}\right)\right| n_{R}: R \in A\right) \\
& =\sum_{\left.\left(b_{1}, \ldots, b_{m}\right) \sim \mid n_{R}: R \in A\right)} P_{\beta}\left[\left(b_{1}, \ldots, b_{m}\right) ; n_{R}, R \in A\right]
\end{aligned}
$$

In the case where $N=\mathscr{N}_{A}=1$, Eq. (3.29) is simply saying that $\left(b_{1}, \ldots, b_{m}\right)$ forms a closed nearest neighbor walk of the type discussed in Section 2. In this case formula (3.31) is similar to (2.20). Equation (3.31) gives a probabilistic representation for the $N$-particle Green's function on the state $\left.\mid n_{R}: R \in A\right)$. If we sum now over $n_{R}, R \in A$, we obtain a probabilistic representation for the partition function of the free Bose gas,

$$
\begin{aligned}
\operatorname{Tr} \exp \left(-\beta \mathscr{H}_{A, B}-h \mathcal{N}_{R}\right)= & \left(\prod_{R \in A} \sum_{n_{R}=0}^{\infty}\right) \exp \left(-h \sum_{R} n_{R}\right) \\
& \times \sum_{\left.\left(b_{1}, \ldots, b_{m}\right) \sim \mid n_{R}: R \in A\right)} P_{\beta}\left[\left(b_{1}, \ldots, b_{m}\right) ; n_{R}, R \in A\right]
\end{aligned}
$$

In the following section we shall see that there is an exactly analogous representation for the Heisenberg partition function.

\section{HEISENBERG MODEL}

For the Heisenberg model we associate with each $R$ in $A$ a spin vector $\bar{S}(R)=\left(S_{x}(R), S_{y}(R), S_{z}(R)\right)$, where the components of $\bar{S}$ are angular momentum operators acting on the irreducible representation of $S U(2)$ with spin $S$. They thus satisfy the commutation relations

$$
\begin{aligned}
& {\left[S_{x}(R), S_{y}\left(R^{\prime}\right)\right]=i \delta_{R R^{\prime}} S_{z}(R)} \\
& {\left[S_{y}(R), S_{z}\left(R^{\prime}\right)\right]=i \delta_{R R^{\prime}} S_{x}(R)} \\
& {\left[S_{z}(R), S_{x}\left(R^{\prime}\right)\right]=i \delta_{R R^{\prime}} S_{y}(R)}
\end{aligned}
$$

and the identity

$$
\bar{S}(R)^{2}=S_{x}(R)^{2}+S_{y}(R)^{2}+S_{z}(R)^{2}=S(S+1)
$$

The Hamiltonian for the Heisenberg model we consider here is

$$
\mathscr{H}_{A}^{S}=\frac{1}{2} \sum_{R \in A, \delta}\left\{(2 S)^{-1}[\bar{S}(R)-\bar{S}(R+\delta)]^{2}-1\right\}
$$


This represents a nearest neighbor interaction and the operator has been normalized to have ground-state energy 0 . The special scaling $(2 S)^{-1}$ has been chosen to simplify formulas and the comparison with the free gas, as will become clear below.

We can consider $\mathscr{H}_{A}^{S}$ defined on the subspace of the free Bose space spanned by the vectors $\left|n_{R}: R \in A\right\rangle$ with $0 \leqslant n_{R} \leqslant 2 S$. The operator $S_{z}\left(R_{0}\right)$ then acts as

$$
S_{z}\left(R_{0}\right)\left|n_{R}: R \in \Lambda\right\rangle=\left(n_{R_{0}}-S\right)\left|n_{R}: R \in A\right\rangle
$$

The number operator restricted to this subspace can thus be written as

$$
\mathscr{N}_{A}=\sum_{R \in A} S_{z}(R)+S
$$

Clearly $\left[\mathscr{H}_{A}^{S}, \mathscr{N}_{A}\right]=0$. The finite-volume free energy is defined as for the Bose gas by

$$
f_{\Lambda, S}(\beta, h)=|\Lambda|^{-1} \log \left[\operatorname{Tr} \exp \left(-\beta \mathscr{H}_{A}^{S}-h \mathscr{N}_{A}\right)\right]
$$

but here the trace is over the Heisenberg subspace. The thermodynamic free energy is

$$
f_{S}(\beta, h)=\lim _{|A| \rightarrow \infty} f_{A, S}(\beta, h)
$$

We want to obtain a random walk representation for the Heisenberg free energy similar to the representation found for the free Bose gas in the previous section. To this end we want to represent $\mathscr{H}_{A}^{S}$ on particle states.

Let $\Lambda_{S}^{N} \subset A^{N}$ be the set

$$
\Lambda_{S}^{N}=\left\{\left(R_{1}, \ldots, R_{N}\right) \in \Lambda^{N} \mid n_{R}\left(R_{1}, \ldots, R_{N}\right) \leqslant 2 S \text { for all } R \in A\right\}
$$

We want to define a transformation $V$ (not necessarily unitary) from the $N$-particle $\left(\mathcal{N}_{\Lambda}=N\right)$ Heisenberg space to $L_{\text {sym }}^{2}\left(A_{S}^{N}\right)$, which can be naturally identified with a subspace of $L_{\text {sym }}^{2}\left(A^{N}\right)$. Then, if $\mathscr{H}_{A, N}^{S}$ denotes the restriction of $\mathscr{H}_{A}^{S}$ to $\mathscr{N}_{A}=N$, we get, with $H_{A, N}^{S}=V \mathscr{H}_{A, N}^{S} V^{-1}$ as in (3.14)

$$
f_{A, S}(\beta, h)=\frac{1}{|A|} \log \left[\sum_{N=0}^{2 S|A|} e^{-h N} \operatorname{Tr}_{L_{\text {sym }}^{2}\left(A_{S}^{N}\right)} \exp \left(-\beta H_{\Lambda, N}^{S}\right)\right]
$$

We want to choose $V$ such that the Green's function $G_{A, N}^{S}\left(\beta, \mathbf{R}, \mathbf{R}^{\prime}\right)$ for $H_{A, N}^{S}$, defined as in (3.15), has a representation as a probability. From Section 2 we know that as a necessary condition $\left(H_{A, N}^{S}\right)^{*}$ must have eigenvalue 0 with corresponding eigenfunction

$$
\psi_{0}\left(R_{1}, \ldots, R_{N}\right)=1, \quad\left(R_{1}, \ldots, R_{N}\right) \in A_{S}^{N}
$$


Thus we want $V^{*}$ to map $\psi_{0}$ to the ground state of $\mathscr{H}_{A}^{S}$ on the subspace $\mathscr{N}_{A}=N$.

The first problem in constructing $V$ is to find this ground state of $\mathscr{H}_{A}^{S}$. This can be done by observing that if we define $S^{ \pm}(R)=S_{x}(R) \pm i S_{y}(R)$, then $\mathscr{H}_{A}^{S}$ commutes with $\sum_{R \in A} S^{+}(R)$. The action of $S^{+}(R)$ is to create a particle at site $R$. The subspace $\mathscr{N}_{A}=0$ consists only of the state $\left|n_{R}=0 ; R \in \Lambda\right\rangle$, which is a ground state of $\mathscr{H}_{A}^{S}$. Thus, the (unnormalized) ground state on $\mathscr{H}_{A}=N$ must be

$$
\begin{aligned}
(N !)^{-1 / 2}\left[\sum_{R \in A} S^{+}(R)\right]^{N}\left|n_{R}=0, R \in A\right\rangle \\
=\sum_{R_{1} \in A} \cdots \sum_{R_{N} \in A} \mathscr{C}\left(R_{1}, \ldots, R_{N}\right)\left|R_{1}, \ldots, R_{N}\right\rangle
\end{aligned}
$$

where $|\mathbf{R}\rangle$ was defined in (3.6) and

$$
\mathscr{C}(\mathbf{R})=\left[\prod_{R \in A} \frac{(2 S) !}{\left[2 S-n_{R}(\mathbf{R})\right] !}\right]^{1 / 2}
$$

and $\mathscr{C}(\mathbf{R})=0$ if $\mathbf{R} \notin \Lambda_{s}^{N}$.

Comparing with (3.10), it is natural to try to define $V^{*}$ by

$$
\begin{aligned}
V^{*}: & L_{\text {sym }}^{2}\left(\Lambda_{S}^{N}\right) \ni \psi \mapsto|\psi\rangle \\
& \equiv \sum_{R_{i} \in A, 1 \leqslant i \leqslant N} \mathscr{C}\left(R_{1}, \ldots, R_{N}\right) \psi\left(R_{1}, \ldots, R_{N}\right)\left|R_{1}, \ldots, R_{N}\right\rangle
\end{aligned}
$$

Notice that clearly $|\psi\rangle$ belongs to the Heisenberg subspace. $\psi_{0}$ is mapped to the ground state for $\mathscr{H}_{A}^{S}$ on $\mathcal{N}_{A}=N$. The operators $V$ and $V^{-1}$ are given by

$$
\begin{gathered}
V:|\varphi\rangle \mapsto \hat{\varphi}(\mathbf{R}) \equiv \mathscr{C}(\mathbf{R})\langle\mathbf{R} \mid \phi\rangle \in L_{\mathrm{sym}}^{2}\left(A_{S}^{N}\right) \\
V^{-1}: \quad \hat{\varphi} \mapsto|\varphi\rangle \equiv \sum_{\mathbf{R} \in \Lambda_{S}^{N}} \mathscr{C}(\mathbf{R})^{-1} \hat{\varphi}(\mathbf{R})|\mathbf{R}\rangle
\end{gathered}
$$

Thus, $V$ is not an isometry. We want to compute the transformed operator $H_{A, N}^{S}=V \mathscr{H}_{\Lambda, N}^{S} V^{-1}$.

Writing

$$
\begin{aligned}
\mathscr{H}_{A}^{S}= & \frac{1}{2} \sum_{R, \delta} \frac{1}{2 S}\left[2 S^{2}-S^{+}(R) S^{-}(R+\delta)-S^{-}(R) S^{+}(R+\delta)\right. \\
& \left.-2 S^{z}(R) S^{z}(R+\delta)\right]
\end{aligned}
$$


an easy calculation gives

$$
\begin{aligned}
\mathscr{H}_{A}^{S}\left|R_{1}, \ldots, R_{N}\right\rangle \\
=\sum_{i=1}^{N} 2 d\left[1-\frac{1}{2 d} \sum_{\delta} \frac{n_{R_{i}+\delta}(\mathbf{R})}{2 S}\right]\left|R_{1}, \ldots, R_{N}\right\rangle \\
\quad-\sum_{i=1}^{N} \sum_{\delta}\left[1-\frac{n_{R_{i}}(\mathbf{R})-1}{2 S}\right]^{1 / 2}\left[1-\frac{n_{R_{i}+\delta}(\mathbf{R})}{2 S}\right]^{1 / 2}\left|R_{1}, \ldots, R_{i}+\delta, \ldots, R_{N}\right\rangle
\end{aligned}
$$

Observing that from (4.12)

$$
\begin{aligned}
\mathscr{C}(\mathbf{R}) & {\left[1-\frac{n_{R_{1}}(\mathbf{R})-1}{2 S}\right]^{1 / 2}\left[1-\frac{n_{R_{1}+\delta}(\mathbf{R})}{2 S}\right]^{1 / 2} } \\
= & {\left[1-\frac{n_{R_{1}}\left(R_{1}+\delta, \ldots, R_{N}\right)}{2 S}\right] \mathscr{C}\left(R_{1}+\delta, \ldots, R_{N}\right) }
\end{aligned}
$$

we get by a straightforward computation using (4.14)-(4.18)

$$
\begin{aligned}
{\left[H_{A, N}^{S} \hat{\phi}\right](\mathbf{R}) } & \\
= & \sum_{i=1}^{N}\left\{\left[\sum_{\delta}\left(1-\frac{n_{R_{i}+\delta}(\mathbf{R})}{2 S}\right)\right] \hat{\phi}(R)\right. \\
& \left.-\sum_{\delta}\left[1-\frac{n_{R_{i}}\left(R_{1}, \ldots, R_{i}+\delta, \ldots, R_{N}\right)}{2 S}\right] \hat{\phi}\left(R_{1}, \ldots, R_{i}+\delta, \ldots, R_{N}\right)\right\}
\end{aligned}
$$

Comparing this expression with Eq. (2.11), we see that indeed $H_{\Lambda, N}^{S}$ defines a process on the lattice $A_{S}^{N}$ with local times defined by

$$
\tau_{S}\left(\mathbf{R}, \mathbf{R}^{\prime}\right) \equiv\left[1-\frac{n_{R_{i}}\left(\mathbf{R}^{\prime}\right)}{2 S}\right]^{-1} \quad \text { for } \quad \mathbf{R}^{\prime}=\left(R_{1}, \ldots, R_{i}+\delta, \ldots, R_{N}\right)
$$

We can also think of the local times as being defined on bonds $b$ on $A$, but then they depend on the particle configuration $\mid n_{R} ; R \in A$ ),

$$
\tau_{S}(b, n) \equiv \tau_{S}(n(b)) \equiv\left[1-\frac{n(\bar{b})}{2 S}\right]^{-1}
$$

where $\bar{b}$ denotes the bond opposite to $b\left(b=\overrightarrow{R^{\prime} R}\right.$ gives $\left.b=\overrightarrow{R R}{ }^{\prime}\right)$ and where we use the notation (3.27).

Definition. We define the spin- $S$ Heisenberg process of $N$ particles on $A$ as the Markov process on $A_{S}^{N}$ defined by local times (4.20) [or (4.21)]. 
According to (4.21) the local time on a bond depends on the number of particles at the site to which the bond points. The local time is greater the more occupied this site is. If the site is filled, the local time is infinity, i.e., there can be no jumps along the bond. The lack of self-adjointness of the generator $H_{A, N}^{S}$ is reflected in the fact that $\tau\left(\mathbf{R}, \mathbf{R}^{\prime}\right) \neq \tau\left(\mathbf{R}^{\prime}, \mathbf{R}\right)$ (except for the case $S=1 / 2$; see below).

Recalling Eq. (4.9), we have proved the following result.

Theorem. If $G_{A, N}^{S}\left(\beta, \mathbf{R}, \mathbf{R}^{\prime}\right)$ denotes the Green's function for the Heisenberg process, then the finite-volume free energy for the Heisenberg model given in (4.6) is

$$
f_{\Lambda, S}(\beta, h)=|\Lambda|^{-1} \log \left[\sum_{N=0}^{2 S|A|} \frac{e^{-h N}}{N !} \sum_{\mathbf{R} \in A_{S}^{N}} \sum_{\pi \in S_{N}} G_{\Lambda, N}^{S}\left(\beta, \mathbf{R}, \mathbf{R}_{\pi}\right)\right]
$$

Equation (4.22) corresponds to (3.21) in the free case.

It is now easy to give the representation corresponding to $(3.32)$ for the Heisenberg model. In analogy with (3.30), we define, for configurations $\mid n_{R} ; R \in A$ ) with $0 \leqslant n_{R} \leqslant 2 S$ for all $R \in A$, the probabilities

$$
\begin{aligned}
P_{\beta}^{S} & {\left[\left(b_{1}, \ldots, b_{m}\right) ; n_{R}, R \in A\right] } \\
= & \int_{0=t_{0}<t_{1}<\cdots<t_{m}<\beta} d t_{1} \cdots d t_{m} \prod_{i=1}^{m} \prod_{b \neq b_{i}} \\
& \times \exp \left\{-\left[n(b)+\sum_{j=1}^{i-1} d b_{j}(b)\right]\left(t_{i}-t_{i-1}\right) / \tau_{S}\left(n(\bar{b})+\sum_{j=1}^{i-1} d b_{j}(\bar{b})\right)\right\} \\
& \times\left(-\frac{d}{d t_{i}}\right) \exp \left\{-\left[n\left(b_{i}\right)+\sum_{j=1}^{i-1} d b_{j}\left(b_{i}\right)\right]\left(t_{i}-t_{i-1}\right) / \tau_{S}\left(n\left(\bar{b}_{i}\right)+\sum_{j=1}^{i-1} d b_{j}\left(\bar{b}_{i}\right)\right)\right\} \\
& \times \prod_{b} \exp \left[-n(b)\left(\beta-t_{m}\right) / \tau_{S}(n(\bar{b}))\right]
\end{aligned}
$$

Notice that if the walk $\left.\left(b_{1}, \ldots, b_{m}\right) \sim \mid n_{R} ; R \in A\right)$ ever accumulates more than $2 S$ particles at a site, the above probability is 0 . We get the representations

$$
\begin{aligned}
\left(n_{R}: R \in A\left|\exp \left(-\beta \mathscr{H}_{A}^{S}\right)\right| n_{R}: R \in A\right) \\
\quad=\sum_{\left.\left(b_{1}, \ldots, b_{m}\right) \sim \mid n_{R} ; R \in A\right)} P_{\beta}^{S}\left[\left(b_{1}, \ldots, b_{m}\right) ; n_{R}, R \in A\right]
\end{aligned}
$$

and thus

$$
\begin{aligned}
\operatorname{Tr} \exp \left(-\beta \mathscr{H}_{A}^{S}-h \mathcal{N}_{A}\right)= & \left(\prod_{R \in A} \sum_{n_{R}=0}^{2 S}\right) \exp \left(-h \sum_{R \in A} n_{R}\right) \\
& \times \sum_{\left.\left(b_{1}, \ldots, b_{m}\right) \sim \mid n_{R} ; R \in A\right)} P_{\beta}^{S}\left[\left(b_{1}, \ldots, b_{m}\right) ; n_{R}, R \in A\right]
\end{aligned}
$$


We can think of (3.32) as being the $S=\infty$ case of (4.25), since then $\tau_{S=\infty}(n(\bar{b}))=1$.

While the probabilities $P_{\beta}$ for the free Bose gas correspond to a diffusion of free identical particles, the probabilities $P_{\beta}^{S}$ correspond to a diffusion of interacting particles, where the interaction is nearest neighbor. The effect of the interaction is to slow down the diffusion. This slowing down is of course expressed in the fact that the local times $\tau_{S} \geqslant 1$. A particle moving with no particles nearby will move freely, since now $\tau_{S}=1$. If there are particles at neighboring sites, $\tau_{S}>1$, then the diffusion slows down. $P_{\beta}^{S}\left[\left(b_{1}, \ldots, b_{m}\right) ; n_{R}, R \in A\right]$ is the probability that the particle gas will return to its original configuration in time $\beta$ having made the moves $b_{1}, \ldots, b_{m}$.

The special case $S=1 / 2$ is illustrative because it is the simplest; each site is either empty or occupied. The local times along all bonds in the lattice $\Lambda_{S=1 / 2}^{N}$ are all equal to 1 , so the generator is self-adjoint in this case. The interaction between particles is due to the restriction from $A^{N}$ to $\Lambda_{S=1 / 2}^{N}$. Another way of expressing this is that the particles move around freely, but reflect off each other as a hard-core gas. The generator for the diffusion is the Laplacian on $\Lambda_{S=1 / 2}^{N}$ with Neumann boundary conditions.

In this gas there will be regions with low density where the particles move almost freely, but there will also be clusters of particles. In the middle of a cluster where the particles are totally surrounded by other particles the diffusion will have slowed down to zero. Stated otherwise, when a cluster first has formed, it can only dissolve or evaporate from the boundary.

At a fixed time the configuration of the gas is like the configuration of a regular Ising model, the cluster being bounded by Peierls contours. The difference from the Ising model is that in the Heisenberg model there is also a time parameter. To find the Heisenberg partition function, we have to sum not only over contours, but over time-periodic contours of period $\beta$. In fact, if we condition the spin- $1 / 2$ Heisenberg expectations to stationary configurations, i.e., no jumps, we get exactly the Ising model. Indeed, let $\sigma(R)=n(R)-1 / 2= \pm 1 / 2$; then from (4.25) the partition function for no jumps is

$$
\begin{aligned}
\left.Z_{S=1 / 2}^{\text {Heisenberg }} \text { (no jumps }\right) & \\
= & \left(\prod_{R \in A} \sum_{n_{R}=0}^{1}\right) \exp \left(-\sum_{R \in A} n_{R}\right) \prod_{b} \exp \left[-\beta n(b) / \tau_{S}(n(\bar{b}))\right] \\
= & \exp [-(\beta d+h)|A| / 2]\left(\prod_{R \in A} \sum_{\sigma_{R}= \pm 1 / 2}\right) \\
& \times \exp \left[-h \sum_{R} \sigma(R)+\beta \sum_{R} \sum_{\delta} \sigma(R) \sigma(R+\delta)\right]
\end{aligned}
$$


Similarly, if we define the spin- $S$ Ising model from the partition function

$$
\begin{aligned}
Z_{\text {Ising }}^{S}(\beta, h)= & \exp [-(\beta d+h)|\Lambda| S] \\
& \times \prod_{R \in A}\left(\sum_{\sigma_{R}=-S}^{S}\right) \exp \left[-h \sum_{R} \sigma(R)+\beta \sum_{R} \sum_{\sigma} \frac{\sigma(R) \sigma(R+\delta)}{2 S}\right]
\end{aligned}
$$

we get the following result.

Theorem. Expectations conditioned to no jumps in the spin- $S$ Heisenberg model are equal to expectations in the Ising model (4.27).

As a simple consequence, we get the following.

\section{Corollary.}

$$
\operatorname{Tr} \exp \left(-\beta \mathscr{H}_{A, S}-h \mathscr{N}_{A}\right) \geqslant Z_{\text {Ising }}^{s}(\beta, h)
$$

\section{CLASSICAL LIMITS}

In this section we will study the classical limits of the representations (3.32) and (4.25). Our aim is to identify the corresponding classical representations. We will see that they are in fact very simple expansions. The classical limit of spin systems was studied in ref. 6 (see also ref. 4), where it was proved that

$$
\lim _{S \rightarrow \infty}\left[f_{S}\left(\frac{\beta}{S}, \frac{h}{S}\right)-\log (2 S+1)\right]=f_{\mathrm{C}}(\beta, h)
$$

where $f_{\mathrm{C}}(\beta, h)$ is the thermodynamic free energy for the classical Heisenberg model, given by the Hamiltonian

$$
\mathscr{H}_{\mathrm{C}, A}=\frac{1}{2} \sum_{R \in A, \delta} \frac{[\bar{S}(R)-\bar{S}(R+\delta)]^{2}}{2}
$$

Here $\bar{S}(R) \in S^{2}$ for each $R \in A$, so that $\mathscr{H}_{\mathrm{C}, A}$ is a function on the space $\left(S^{2}\right)^{A}$ of functions from $\Lambda$ to $S^{2}$.

The finite-volume free energy is

$$
f_{\mathrm{C}, \lambda}(\beta, h)=\frac{1}{|A|} \log \left\{\left(\prod_{R \in A} \frac{1}{4 \pi} \int_{S^{2}} d S(R)\right) \exp \left[-\beta \mathscr{H}_{\mathrm{C}, A}-h \sum_{R}\left(S_{z}(R)+1\right)\right]\right.
$$


The classical limit stated in terms of thermodynamic free energies in (5.1) of course also holds in a finite volume.

The corresponding limit of the free Bose gas (3.3)-(3.5) is given by the Gaussian model,

$$
\mathscr{H}_{\mathrm{G}, A}=\frac{1}{2} \sum_{R \in A, \delta}|z(R+\delta)-z(R)|^{2}
$$

where $z(R) \in \mathbb{C}, R \in \Lambda$, and $\mathscr{H}_{\mathbb{G}, A}$ is defined on $\mathbb{C}^{A}$. The finite-volume free energy is

$$
f_{\mathrm{G}, A}(\beta, h)=\frac{1}{|A|} \log \left\{\left(\prod_{R \in A} \frac{1}{\pi} \int_{\mathbb{C}} d^{2} z(R) \exp \left[-\beta \mathscr{H}_{\mathrm{G}, \Lambda}-h \sum_{R}|z(R)|^{2}\right]\right\}\right.
$$

The result corresponding to (5.1) is

$$
\lim _{\varepsilon \rightarrow 0}\left[f_{\mathrm{B}}(\varepsilon \beta, \varepsilon h)+\log \varepsilon\right]=f_{\mathrm{G}}(\beta, h)
$$

Since both sides of (5.6) are explicitly computable by going to Fourier variables, this limit is very simple to prove.

We first turn to the representation (3.26) to see what happens to this expansion in the limit (5.6).

Thus we want to study

$$
\lim _{\varepsilon \rightarrow 0}|A|\left(f_{\mathrm{B}, A}(\varepsilon \beta, \varepsilon h)+\log \varepsilon\right)=\lim _{\varepsilon \rightarrow 0}\left(\sum_{j=1}^{\infty} \frac{e^{-j h}}{j} \sum_{R \in A} G_{\Lambda}(j \varepsilon \beta, R, R)+\log \varepsilon\right)
$$

Using the formula (2.18) [see also (2.6)], we get that the right-hand side of $(5.7)$ is

$$
\lim _{\varepsilon \rightarrow 0}\left(\sum_{l=0}^{\infty} \frac{1}{l !} \sum_{R \in A} A^{l}(R, R) \sum_{j=1}^{\infty} \frac{e^{-(2 d \beta+h) \varepsilon j}}{j}(2 d \beta \varepsilon j)^{l}+\log \varepsilon\right)
$$

If $l \neq 0$, we find

$$
\begin{aligned}
\lim _{\varepsilon \rightarrow 0} \sum_{j=1}^{\infty} \frac{e^{-(2 d \beta+h) \varepsilon j}}{j}(2 d \beta \varepsilon j)^{l} & =\int_{0}^{\infty} \frac{e^{-(2 d \beta+h) t}(2 d \beta t)^{l}}{t} d t \\
& =\left(1+\frac{h}{2 d \beta}\right)^{-l}(l-1) !
\end{aligned}
$$

For the $l=0$ term in $(5.8)$ we get

$$
\begin{aligned}
\lim _{\varepsilon \rightarrow 0}\left(\sum_{j=1}^{\infty} \frac{e^{-(2 d \beta+h) \varepsilon j}}{j}+\log \varepsilon\right) & =\lim _{\varepsilon \rightarrow 0}\left[-\log \left(1-e^{-(2 d \beta+h) \varepsilon}\right)+\log \varepsilon\right] \\
& =-\log (2 d \beta+h)
\end{aligned}
$$


Since the left-hand side of (5.7) is identical to the finite-volume free energy for the Gaussian, we arrive, by a simple interchange of limits, at the following random walk expansion:

$$
\exp \left[|A| f_{\mathrm{G}, A}(\beta, h)\right]=(2 d \beta+h)^{-|A|} \exp \left[\sum_{l=1}^{\infty} \frac{1}{l}\left(1+\frac{h}{2 d \beta}\right)^{-l} \sum_{R \in A} A^{l}(R, R)\right]
$$

Equation (5.11) can be interpreted as saying that a Gaussian system is a perfect gas of random loops.

The expansion can be adapted to non-Gaussian systems, but in this case the loops interact. Such an expansion has been studied by Brydges et al. ${ }^{(3)}$

Before going to the classical limit of Heisenberg model, we will also consider the limit of the expansion in (3.32). We get from (3.30)

$$
\begin{aligned}
& \lim _{\varepsilon \rightarrow 0} \exp \left\{|A|\left[f_{\beta, A}(\varepsilon \beta, \varepsilon h)+\log \varepsilon\right]\right\} \\
&=\lim _{\varepsilon \rightarrow 0} \varepsilon^{|A|}\left(\prod_{R \in A} \sum_{n_{R}=0}^{\infty}\right) \exp \left(-h \sum_{R} \varepsilon n_{R}\right) \\
& \quad \times \sum_{\left.\left(b_{1}, \ldots, b_{m}\right) \sim \mid n_{R}: R \in A\right)} P_{\varepsilon \beta}\left[\left(b_{1}, \ldots, b_{m}\right) ; n_{R}, R \in A\right] \\
&=\lim _{\varepsilon \rightarrow 0}\left(\prod_{R \in A} \sum_{n_{R}=0}^{\infty} \varepsilon\right) \exp \left(-h \sum_{R} \varepsilon n_{R}\right) \\
& \times \sum_{\left.\left(b_{1}, \ldots, b_{m}\right) \sim \mid n_{R}: R \in A\right)} \prod_{i=1}^{m} \varepsilon\left[n\left(b_{i}\right)+\sum_{j=1}^{i-1} d b_{j}\left(b_{i}\right)\right] \prod_{b} \exp [-n(b) \varepsilon \beta] \\
&= \prod_{R \in A}\left[\frac{1}{\pi} \int_{\mathbb{C}} d^{2} z(R)\right] \exp \left(-h \sum_{R}|z(R)|^{2}\right] \\
& \times \sum_{\substack{\left(b_{1}, \ldots, b_{m}\right) \\
\text { closed loops }}} \prod_{i=1}^{m}\left|z\left(b_{i}\right)\right|^{2} \prod_{b} \exp \left[-|z(b)|^{2} \beta\right]
\end{aligned}
$$

Here we have adopted the notation $z(b)=z\left(R^{\prime}\right)$ for $b=\overrightarrow{R^{\prime} R}$. Notice that in the classical limit the only compatibility condition on $\left(b_{1}, \ldots, b_{m}\right)$ is that they form closed loops.

It should now be clear that the expansion (5.12) is what one would get from writing $z=|z| e^{i \theta}$ and then expanding the part of the exponential in (5.5) containing terms with $e^{i \theta}$. The expansion contains only closed loops because the only nonzero terms after integration are those that are real.

We shall now see in a very similar manner that the classical limit of 
the representation (4.25) for the Heisenberg model is what we would get by writing (5.2) in terms of

$$
S^{ \pm}(R)=S_{x}(R) \pm i S_{y}(R)=\left[1-S_{z}^{2}(R)\right]^{1 / 2} \exp [ \pm i \theta(R)]
$$

The part of the exponential in (5.3) containing terms with $S^{ \pm}(R)$ is then expanded and the $\theta(R)$ variables integrated out just as in the Gaussian case. It is again clear that the sum over sequences of bonds is restricted to those forming closed loops.

We should emphasize here that this random walk representation is not the same as the Symanzik representation ${ }^{(3)}$ for the classical Heisenberg model. The Symanzik representation is rotation invariant, whereas the above representation depends on the direction of the $S_{z}$ axis. It would be of interest to find a representation for the quantum Heisenberg model which converges to the Symanzik representation in the classical limit.

From (5.1) the appropriate limit is

$$
\begin{aligned}
& \lim _{S \rightarrow \infty}(2 S+1)^{-|\Lambda|} \operatorname{Tr} \exp \left(\frac{-\beta}{S} \mathscr{H}_{A}^{S} \frac{-h}{S} \mathscr{N}_{A}\right) \\
& =\lim _{S \rightarrow \infty} \prod_{R \in A}\left(\frac{1}{2 S+1} \sum_{n_{R}=0}^{2 S}\right) \exp \left(-h \sum_{R} \frac{n_{R}}{S}\right) \\
& \times \sum_{\left.\left(b_{1}, \ldots, b_{m}\right) \sim \mid n_{R} ; R \in A\right)} P_{\beta / S}^{S}\left[\left(b_{1}, \ldots, b_{m}\right) ; n_{R}, R \in A\right] \\
& =\lim _{S \rightarrow \infty} \prod_{R \in A}\left(\frac{1}{2 S+1} \sum_{n_{R}=0}^{2 S}\right) \exp \left(-h \sum_{R} \frac{n_{R}}{S}\right) \\
& \times \sum_{\left(b_{1}, \ldots, b_{m}\right) \sim \mid n_{R} ; R \in A} \prod_{i=1}^{m}\left(\frac{n\left(b_{i}\right)}{S}+\sum_{j=1}^{i-1} \frac{d b_{j}\left(b_{i}\right)}{S}\right) \\
& \times\left[1-\frac{n\left(\bar{b}_{i}\right)+\sum_{j=1}^{i-1} d b_{j}\left(\bar{b}_{i}\right)}{2 S}\right] \prod_{b} \exp \left\{-\frac{n(b)}{S} \beta\left[1-\frac{n(\bar{b})}{2 S}\right]\right\} \\
& =\prod_{R \in A} \frac{1}{2} \int_{-1}^{1} d S_{z}(R) \exp \left\{-h \sum_{R}\left[S_{z}(R)+1\right]\right\} \\
& \times \exp \left\{-\frac{\beta}{2} \sum_{R} \sum_{\delta}\left[1-S_{z}(R) S_{z}(R+\delta)\right]\right\} \\
& \times \sum_{\begin{array}{c}
\left(b_{1}, \ldots, b_{m}\right) \\
\text { closed loops }
\end{array}} \prod_{i=1}^{m} \frac{1}{2}\left[S_{z}\left(b_{i}\right)+1\right]\left[1-S_{z}\left(\bar{b}_{i}\right)\right]
\end{aligned}
$$

Since $S^{+}(R) S^{-}(R)=1-S_{z}(R)^{2}$, we see that this random walk representation is indeed an expansion of the exponential containing terms with $S^{ \pm}$. 


\section{ACKNOWLEDGMENTS}

This work was partially supported by NSF grants DMS 8900244 (J.G.C.) and DMS 9002416 (J.P.S.).

\section{REFERENCES}

1. J. Bricmont, Inequalities between different classical spin models, Phys. Lett. 57A:411-413 (1976).

2. J. Bricmont, L. Lebowitz, and C. E. Pfister, Low temperature expansion for continuous spin Ising models, Commun. Math. Phys. 78:117-135 (1980).

3. D. Brydges, J. Fröhlich, and T. Spencer, The random walk representation of classical spin systems and correlation inequalities, Commun. Math. Phys. 83:123-150 (1982).

4. J. Conlon and J. P. Solovej, On asymptotic limits for the quantum Heisenberg model, J. Phys. A: Math. Gen. 23:3199-3213 (1990).

5. F. Dyson, General theory of spin-wave interactions, Phys. Rev. 102:1217-1230 (1956).

6. E. Lieb, The classical limit of quantum spin systems, Commun. Math. Phys. 31:327-340 (1973).

7. B. Simon, The $P\left(\varphi_{2}\right)$ Euclidean (Quantum) Field Theory (Princeton University Press, 1974).

8. C. Thompson, A vector spin correlation inequality, Phys. Lett. 43A:259-260 (1973). 